\title{
Inorganic polyphosphate adsorbed onto hydroxyapatite for guided bone regeneration: An animal study
}

\author{
Kazuya DOI ${ }^{1}$, Takayasu KUBO ${ }^{1}$, Ryou TAKESHITA¹, Shiho KAJIHARA ${ }^{1}$, Shinichi KATO², Yumi KAWAZOE², \\ Toshikazu SHIBA² and Yasumasa AKAGAWA ${ }^{1}$ \\ ${ }^{1}$ Department of Advanced Prosthodontics, Hiroshima University Graduate School of Biomedical and Health Sciences, 1-2-3, Kasumi, Minami-ku, \\ Hiroshima 734-8553, Japan \\ ${ }^{2}$ Regenetiss Incorporation, 2-24-16, Nakamachi, Koganei, Tokyo 184-0012, Japan \\ Corresponding author, Kazuya DOl; E-mail: kazuya17@hiroshima-u.ac.jp
}

\begin{abstract}
Inorganic polyphosphate $(\operatorname{poly}(\mathrm{P}))$ is recognized as a therapeutic agent that promotes fibroblast growth factor and enhances osteogenic differentiation, and in vivo, when adsorbed onto interconnected porous calcium hydroxyapatite (IP-CHA) enhances bone regeneration. The present study focused on the effect of poly(P) adsorbed onto IP-CHA granules (Poly(P)/IP-CHA) in guided bone regeneration (GBR). Dental implants were placed into the edentulous mandibular areas of five Beagle-Labrador hybrid dogs with screw expose on the buccal side, and then bone defects were filled Poly(P)/IP-CHA (test) or IP-CHA (control). After 12 weeks, histological evaluation and histomorphometrical analysis were performed. Newly-bone formation around exposed implant screw was clearly detected in the test-group. The ratio for regenerated bone height in the test group versus the control-group was $85.6 \pm 20.2$ and $62.6 \pm 23.8$, respectively, with no significant difference, while, that for bone implant contact was significantly higher (67.9 \pm 11.8 and $48.8 \pm 14.1$, respectively). These findings indicate that Poly(P)/IP-CHA enhances bone regeneration in GBR.
\end{abstract}

Keywords: Polyphosphate, Bone regeneration, Hydroxyapatite, IP-CHA, Guided bone regeneration

\section{INTRODUCTION}

Dental implant therapy has been used to replace missing teeth. Modern prosthetic implants usually achieve optimal esthetic and functional recovery, as successfully evaluated by contemporary criteria. In certain clinical situations, however, it is difficult to perform implant therapy because of insufficient bone at the implant site. After tooth extraction, new bone grows in the bone socket but does not completely restore the original bone shape ${ }^{1)}$. Several reports have also documented reduction in buccal bone volume following tooth extraction, resulting in recession of the mucosal margins following remodeling of the alveolar ridge ${ }^{2,3)}$. In such cases, bone augmentation with graft materials is required for successful implant therapy. Guided bone regeneration (GBR) is an effective method to augment the existing bone at the implant site, and it is most commonly used to treat mandible bone areas with insufficient bone volume ${ }^{4,5,6)}$. Autogenous bone is the gold standard as the graft material for GBR, but harvesting surgery may not yield sufficient bone for grafting and can cause persistent pain, nerve damage, fracture, or cosmetic defects at the donor site ${ }^{7,8)}$. Therefore, developing artificial bone graft materials or scaffolds to facilitate new bone growth is anticipated.

Inorganic polyphosphate $(\operatorname{poly}(\mathrm{P}))$, one of the biological polymer, and the basic fibroblast growth factor (bFGF) act synergistically to promote bone formation. Shiba et al. demonstrated that poly $(\mathrm{P})$

Color figures can be viewed in the online issue, which is available at J-STAGE.

Received Oct 2, 2013: Accepted Dec 12, 2013

doi:10.4012/dmj.2013-275 JOI JST.JSTAGE/dmj/2013-275 enhances the activity of bFGF on osteoblastic cells and increased calcification, possibly by stabilizing bFGF receptor expression ${ }^{9}$. Furthermore, poly $(\mathrm{P})$ enhanced the differentiation of human mesenchymal stem cells into osteoblastic cells at the early and later stages of osteoblast differentiation ${ }^{10)}$. On the basis of these preclinical studies suggesting that poly $(\mathrm{P})$ promotes cell proliferation and osteogenic differentiation, we have been developing bone graft biomaterials containing poly $(\mathrm{P})$ for accelerated bone regeneration. In our previous studies, we demonstrated that blocks of interconnected porous calcium hydroxyapatite (IP$\mathrm{CHA}^{11)}$ with adsorbed poly $(\mathrm{P})$ accelerated bone formation $^{12,13)}$. However, blocks of graft material are difficult to apply in clinical GBR because the topology of the dental bone defect caused by surgical resection and subsequent inflammation is complex and not predictable before treatment. For clinical applications, therefore, smaller granular biomaterials may be superior for implant and periodontal therapies. It is possible to fabricate optimal granule type ${ }^{14}$, and it expects to adsorb poly $(\mathrm{P})$ as well as the block type.

This study was designed to evaluate poly $(\mathrm{P})$ adsorption onto IP-CHA granules for potential GBR application around dental implants.

\section{MATERIALS AND METHODS}

Fabrication of granular Poly $(P) / I P-C H A$ poly $(\mathrm{P})$ sodium salt with an average chain length of 65 phosphate residues (Regenetiss Inc, Nagano, Japan) was prepared. IP-CHA granules (diameter: approximately 

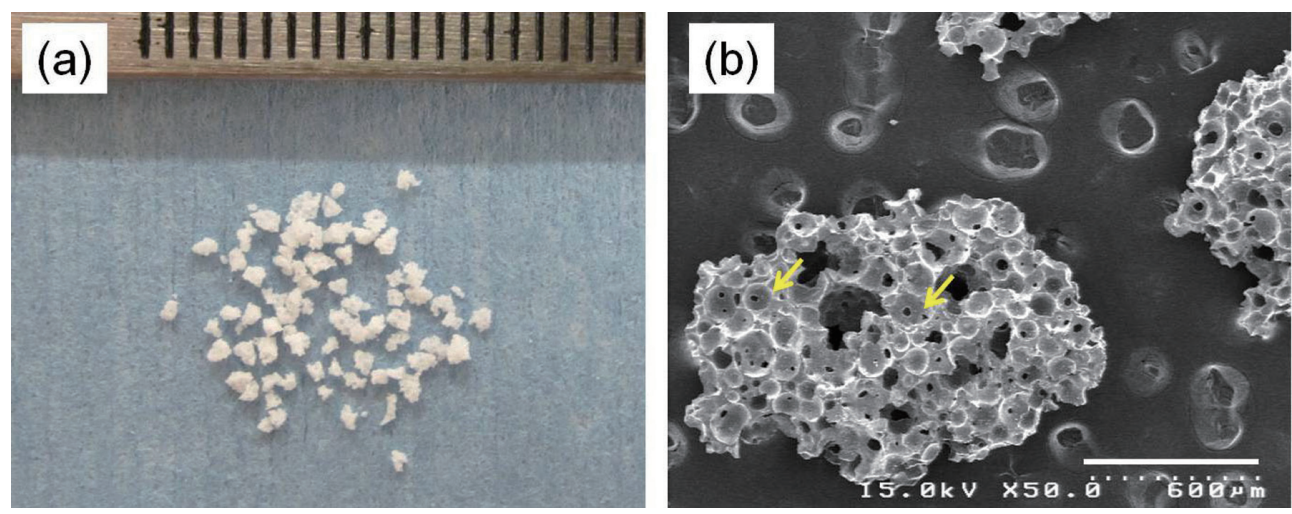

Fig. 1 IP-CHA granule.

(a) The size of the granules was nearly at $0.5 \mathrm{~mm}$ diameter.

(b) SEM image of IP-CHA granule. Almost of pores were connected through interconnective pores (Arrows indicated). The scale bar indicates $600 \mu \mathrm{m}$.

$500 \mu \mathrm{m})\left(\mathrm{NEOBONE}^{\circledR}\right.$, Covalent Materials Corporation, Tokyo, Japan) with $75 \%$ porosity, mean pore diameter of $150 \mu \mathrm{m}$, and 40- $\mu \mathrm{m}$ diameter channels interconnecting pores were used as a scaffold for poly(P) (Fig. 1). To fabricate IP-CHA with adsorbed poly $(\mathrm{P})$ (Poly $(\mathrm{P}) /$ IP-CHA), the IP-CHA granules were soaked in a $25 \% \operatorname{poly}(\mathrm{P})$ solution $(\mathrm{w} / \mathrm{w})$ for $24 \mathrm{~h}$. The solution was then deaerated for $2 \mathrm{~h}$ with an aspirator. Insoluble Poly(P)/IP-CHA precipitate was removed by centrifugation $(2,500 \mathrm{rpm}, 2 \mathrm{~min})$ and dried for at least 3 days at $37^{\circ} \mathrm{C}$. The isolated Poly(P)/IP-CHA material was then sterilized using gamma irradiation.

\section{Evaluation of the Poly $(P) / I P-C H A$ granule structure}

The outer structures of IP-CHA and Poly(P)/IP-CHA granules were observed using a scanning electron microscope (JMS-7300, Nihon Denshi Oyo Co Ltd, Tokyo, Japan) at $\times 100$ and $\times 1,000$ magnification.

Compression tests were performed using Autograph (AUTOGRAPH AGS-X 5kN, Shimazu Seisakusho, Japan). The compression speed was 0.1 $\mathrm{mm} / \mathrm{min}$. Testing was performed vertically toward the granule of IP-CHA and Poly(P)/IP-CHA $(n=6)$.

\section{Evaluation of Poly $(P)$ adsorption}

Both recovered Poly(P)/IP-CHA and IP-CHA were soaked in a $0.05 \%$ toluidine blue solution (w/v) to assess poly $(\mathrm{P})$ adsorption. The mixture was deaerated for 30 min, and the stained grafting material recovered by three rounds of centrifugation (each at 2,000 rpm for 5 min). The stained Poly(P)/IP-CHA or IP-CHA was then dried at room temperature for $24 \mathrm{~h}$ or more.

\section{Evaluation of Poly $(P) / I P-C H A$ for GBR in vivo}

This study was approved by the Research Facilities Committee for Laboratory Animal Science at the Hiroshima University School of Medicine, Hiroshima, Japan. All surgeries were performed under general anesthesia, and all efforts were made to minimize suffering of animals during the experimental period.

The implants used in this study were fabricated from commercially pure titanium and measured $3.3 \mathrm{~mm}$ in diameter and $10 \mathrm{~mm}$ in length (Brånemark ${ }^{\circledR}$ Tiunite MkIII, Nobel Biocare Japan Inc, Tokyo, Japan). Five male Beagle-Labrador hybrid dogs (weighing 20-23 kg and 18-20 months of age) were fed in their cages for 1 month to allow them to acclimate to the environment. After 1 month, right mandibular premolars (P1-P4) were extracted, and the sites were allowed to heal for 3 months prior to implant placement. The surgical procedures were performed under general anesthesia with sodium pentobarbital $(10 \mathrm{mg} / \mathrm{kg})$ and local infiltration anesthesia with $2 \%$ lidocaine and 1:80,000 noradrenaline. Implant placement was performed as follows. A mucoperiosteal flap around the implant site was raised carefully to minimize damage to the underlying periosteum. The residual bone ridge was filed using a grafting blade (MX-grafter ${ }^{\circledR}$, Maxilon Laboratories Inc, Hollis, USA) such that the implant shoulder was level with the buccolingual crestal bone. Two insertion sockets of the implant (3 $\mathrm{mm}$ in diameter and $10 \mathrm{~mm}$ in depth) were prepared in the edentulous section of the mandible using a special electric engine (Nobel Biocare Japan Inc, Tokyo, Japan) with serial cutting drills and a screw tap (Nobel Biocare Japan Inc) in accordance with the Brånemark ${ }^{\circledR}$ system manual. To minimize damage to bone tissue, low speed (maximum $800 \mathrm{rpm}$ ), low pressure on the cutting drill, and continuous external saline irrigation were used. After preparation of the bone socket for implant placement, a buccal bone defect ( $5 \mathrm{~mm}$ in width and $4 \mathrm{~mm}$ in depth) was made in each bone socket to expose the upper 4 screw threads of the implant, and implants then were placed into the bone sockets. Either Poly(p)/IP-CHA (test) or IP-CHA (control) was grafted into the bone defect around the fenestrated implant exposure site $(n=5)$. After applying expanded polytetrafluoroethylene membrane (Gore-Texs, W.L. Gore \& Associates, 

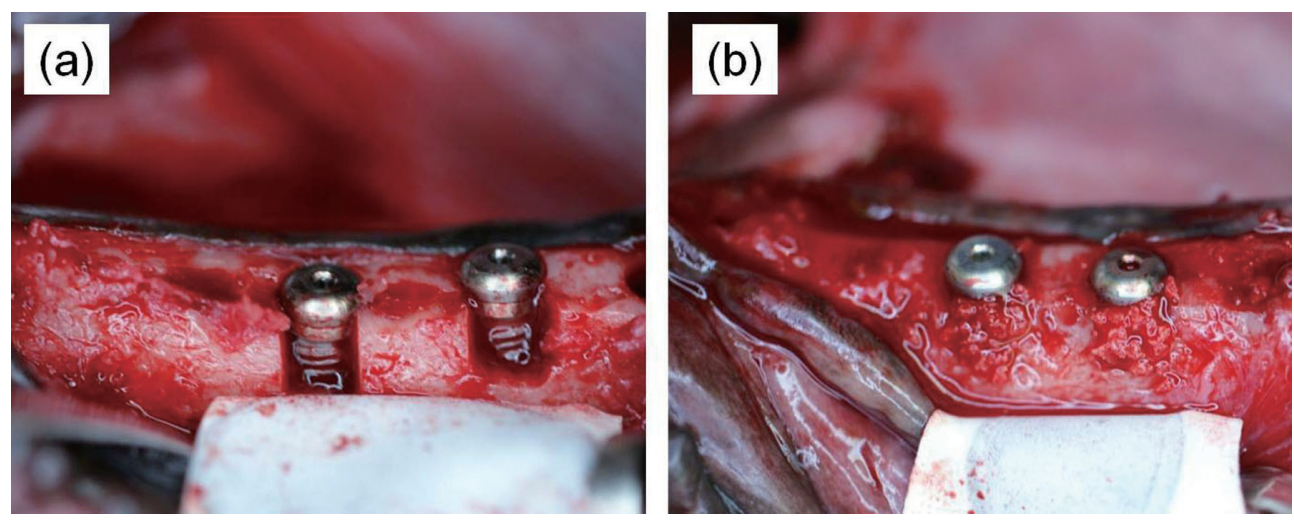

Fig. 2 Implants placed into the defects and granules of Poly(P)/IP-CHA and IP-CHA were filled to cover all exposed screws of implants, then covered with e-PTFE membrane, and fixed with screw pins.

Flagstaff, AZ, USA) (Fig. 2), the mucoperiosteal flap was closed in layers using 4-0 non-absorbable thread (Gore suture, W. L. Gore \& Associates). After 12 weeks, animals were anesthetized and perfused with $10 \%$ neutral formalin through the aorta.

The right mandibles were removed, and tissue blocks containing implants were harvested. The blocks were fixed in $10 \%$ formalin neutral buffer solution for 1 week. After final fixation, these undecalcified blocks were trimmed and dehydrated using ascending concentrations of ethanol and embedded in methyl methacrylate. The resin blocks were cut longitudinally with a half cut of the implant using special thin section equipment. Each block was cut buccolingually to produce $200-\mu \mathrm{m}$ thick sections. Around the implant sites, sections were reduced to $45-\mu \mathrm{m}$ thick using a microgrinding machine (MG-400 CS, EXAKT, Oklahoma City, OK, USA).

Specimens were stained with toluidine blue, images of bone regeneration were digitized, and histomorphometric parameters measured with Image J (National Institutes of Health, Bethesda, Maryland, USA). Ratios of regenerated bone height and bone implant contact (BIC) were measured from the lowest point of the experimentally prepared defect to the highest point of the regenerated bone (Fig. 3).

\section{Statistical analysis}

Obtained data; compressive strength and ratios of regenerated bone height and BIC, were expressed as means $+/-$ standard deviations. The values were statistically analyzed using Student's $t$-tests with the significance level set at $5 \%$.

\section{RESULTS}

Structure of IP-CHA and Poly $(P) / I P-C H A$

Figure 4 compares the structures of Poly(P)/IP-CHA and IP-CHA as revealed by SEM images. Similar to IPCHA, the Poly(P) /IP-CHA granules possessed pores of
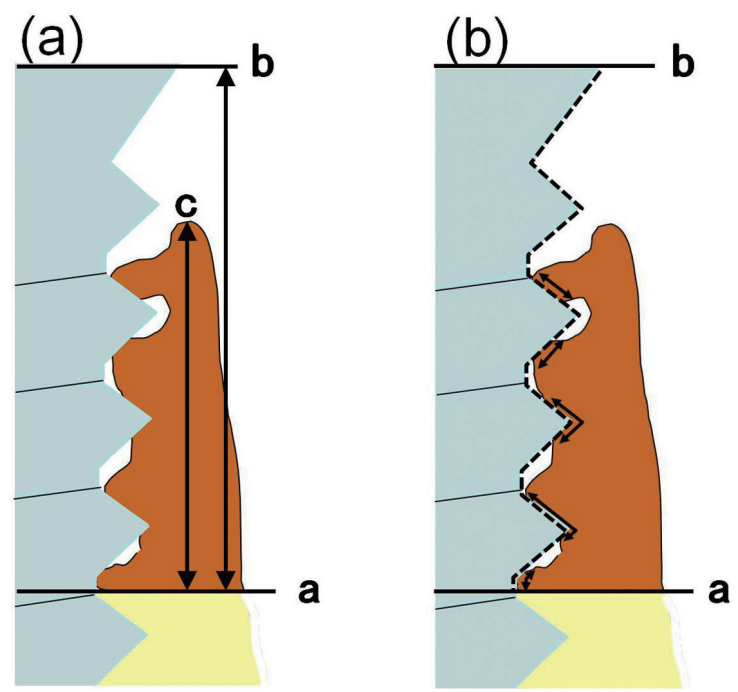

Fig. 3 Schema of histomorphometrical analysis.

The ratio of regenerated bone height was measured as the ratio of bone height regenerated bone $(\mathrm{a}-\mathrm{c})$ in total measured as the distance (c) between the bottom of the experimentally prepared defect (a) and the top of the regenerated bone (b).

The ratio of bone implant contact (BIC) was measured as the ratio of contact length of newly formed bone in total length from the bottom of detect to the top of implant shoulder part.

relatively uniform size that were still interconnected; not sealed with poly $(\mathrm{P})$. The compressive strength were $1709.32 \pm 211.6 \mathrm{mN}$ in the IP-CHA and $1856.48 \pm 55.1$ $\mathrm{mN}$ in the Poly(P)/IP-CHA (Table 1). There was no significant difference between IP-CHA and Poly(P)/IPCHA granules. 

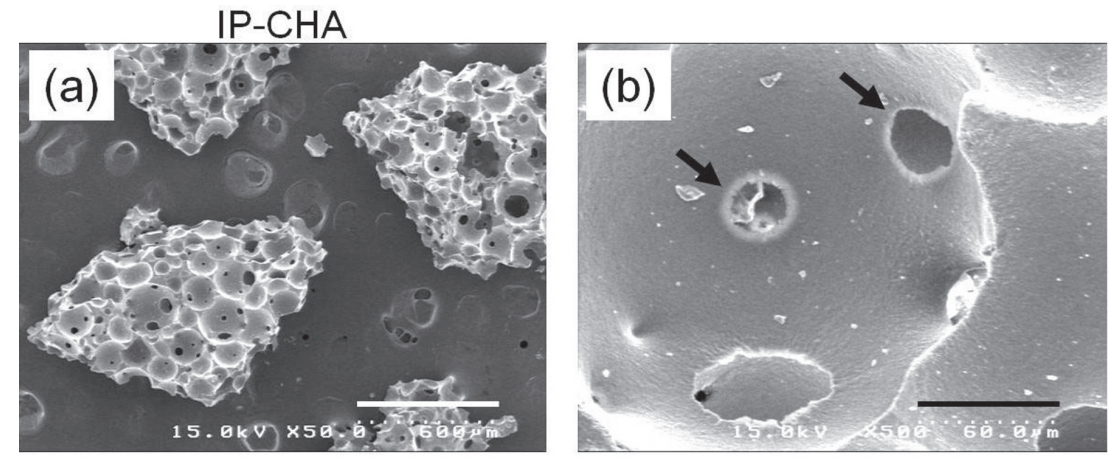

Poly(P)/IP-CHA
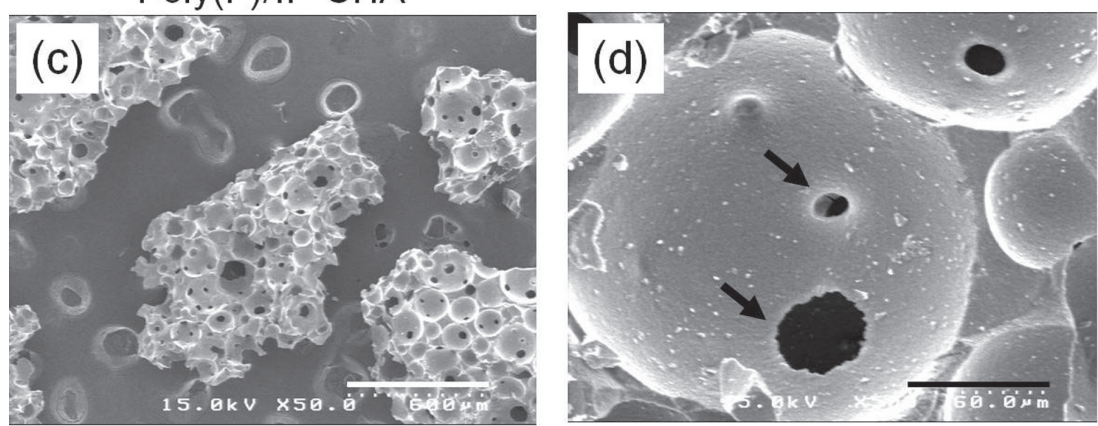

Fig. 4 SEM image of IP-CHA (a, b) and Poly(P)/IP-CHA (c, d).

(a, c): At low magnification. The scale bar indicates $600 \mu \mathrm{m}$.

(b, d): At high magnification. Interporous connections were clearly maintained (Allow indicated). The scale bar indicates $60 \mu \mathrm{m}$.

Table 1 Compressive strength of granule IP-CHA and granule Poly(P)/IP-CHA

\begin{tabular}{lc}
\hline \multicolumn{1}{c}{ Materials } & Compressive strength (SD) \\
\hline IP-CHA & $1709.32(211.6) \mathrm{mN}$ \\
Poly(P)/IP-CHA & $1856.48(55.1) \mathrm{mN}$ \\
\hline
\end{tabular}

$\mathrm{SD}$, standard deviation.

\section{Poly $(P)$ adsorption}

The Poly(P)/IP-CHA granules were stained over the entire surface by the cationic dye toluidine blue, confirming widely distributed adsorption of poly $(\mathrm{P})$, while IP-CHA granules remained unstained (Fig. 5).

\section{Histological and histomorphometric analyses}

All implants healed uneventfully and remained stable throughout the experimental period. No suppuration or abscess formation was observed around any implant, and no membrane exposure was found. Regenerated bone was detected along the set membrane both in implant sites treated with Poly(P)/IP-CHA and those treated with IP-CHA. Furthermore, the original shape of the bone lesions was reconstructed. Bone formation was detected in the bottom portion on the existed bone site, but it was not detected in the pores at central and upper portion of IP-CHAs. Around exposed screws, newly formed bone contacted the implant surface and osseointegration was achieved (Figs. 6a, 6c). Compared with the IP-CHA group, the exposed screws of the Poly(P)/IP-CHA group were well covered by newly formed bone (Figs. 6b, 6d).

The ratio of regenerated bone height was $85.6 \pm 20.2 \%$ in the Poly(P)/IP-CHA group and $62.6 \pm 23.8 \%$ in the IPCHA group (Fig. 7a), but the difference did not reach statistical significance. In contrast, the BIC value was significantly higher in the Poly(P)/IP-CHA group $(67.9 \pm 11.8 \%)$ than that in the IP-CHA group $(48.8 \pm 14.1 \%)$ $(p=0.048)$ (Fig. 7b).

\section{DISCUSSION}

The results of this study indicate that granular Poly $(\mathrm{P}) /$ IP-CHA can accelerate bone regeneration around dental implants with fenestrated defects compared to IP-CHA, and therefore may be a superior bone graft material for GBR. Poly $(\mathrm{P})$ both promotes osteogenic differentiation of stem cells and increases bFGF activity ${ }^{10)}$. Enhanced proliferation of stem cells and differentiation of osteoblasts by poly(P) as well as improved vascularization during the bone healing process may facilitate bone growth. These results confirm and extend previous observations showing that poly $(\mathrm{P})$ adsorbed 

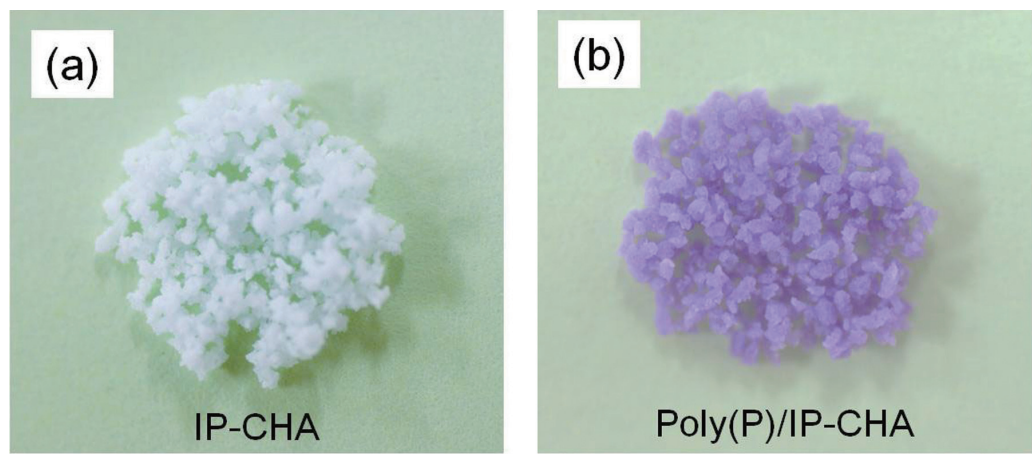

Fig. 5 Chromatic figures of granule type of (a) IP-CHA and (b) Poly(P)/IP-CHA stained with toluidine blue.
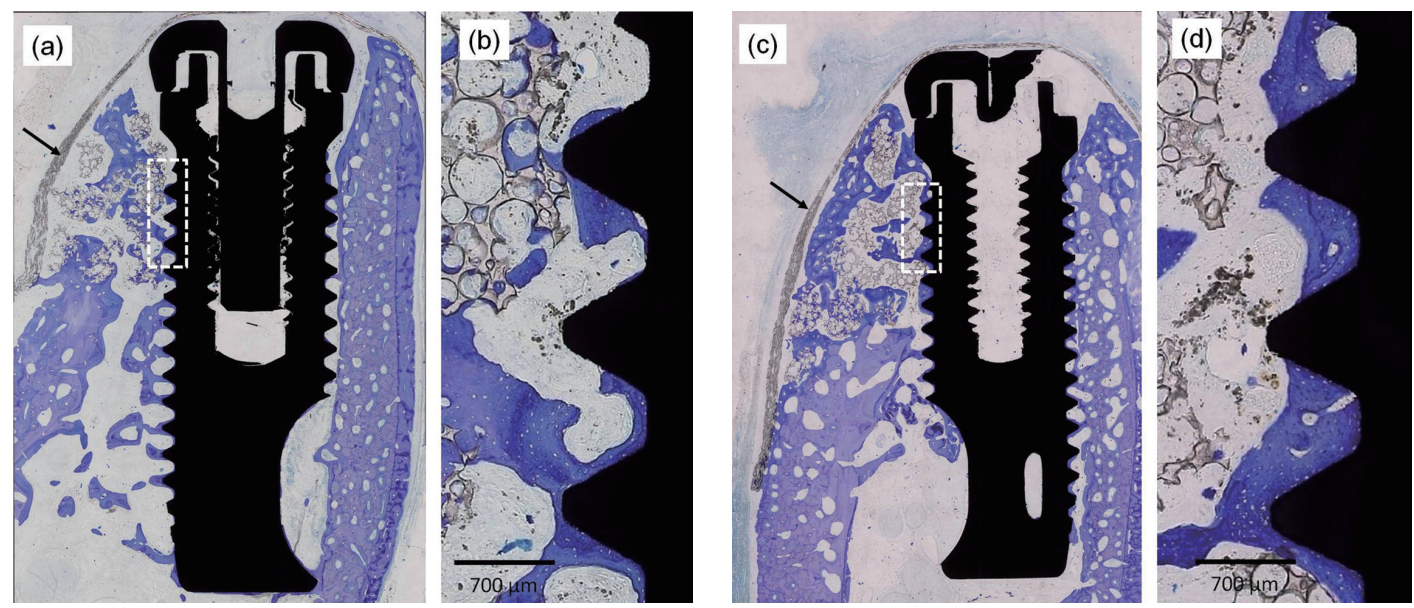

Fig. 6 Histological specimens of IP-CHA $(a, b)$ and Poly $(\mathrm{P}) / \operatorname{IP}-\mathrm{CHA}(\mathrm{c}, \mathrm{d})$.

$(\mathrm{a}, \mathrm{c})$ : Regenerated bone detected at implant shoulder of marginal area. In the both groups, the defects were filled bone, fibrous tissue and graft material, and defect space was reconstructed (Arrows indicated e-PTFE membrane). Bone formation was detected in the bottom portion on the existed bone site, but it was not detected in the pores at central and upper portion of IP-CHAs.

(b, d): Newly formed bone was detected around the exposed screw. High levels of new bone formation were observed in Poly(P)/IP-CHA.

(a)

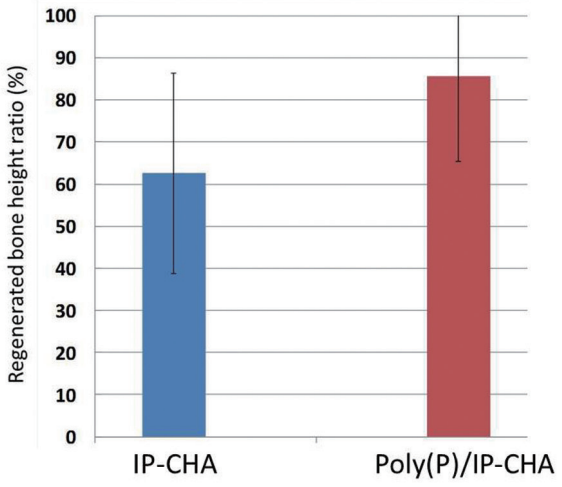

(b)

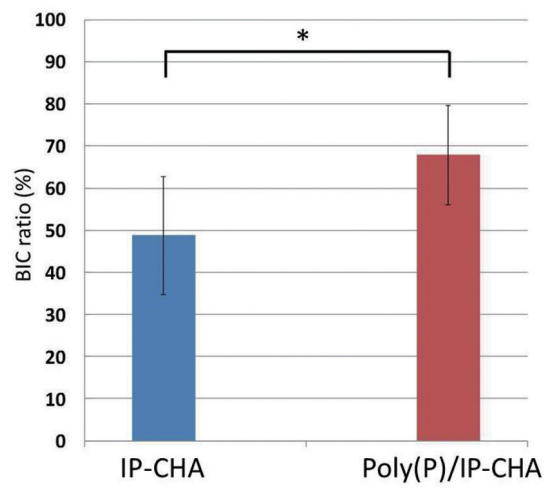

Fig. 7 (a): The ratio of regenerated bone height was $85.6 \pm 20.2 \%$ in the poly(P)/IP-CHA group and $62.6 \pm 23.8 \%$ in the IP-CHA group. There was no significant difference between the groups. (b): The ratio of BIC was $67.9 \pm 11.8 \%$ in the Poly(P)/IP-CHA; this was significantly higher than $48.8 \pm 14.1 \%$ in the IP-CHA $(*: p<0.05)$. 
onto block type IP-CHA composites accelerated early bone regeneration in a rabbit femur model ${ }^{12,13)}$. Blocks of bone graft material are difficult to apply for implant GBR because of the complex topography of the bone defect at the implant site. In this study, we used IP-CHA granules as the scaffold material to adsorbed poly $(\mathrm{P})$. In our previous study, different shapes of granular porous HA were used in mandibular bone socket defects for bone regeneration. The high fill density of granule biomaterials indicated good bone formation ${ }^{14)}$. The 300-500 $\mu \mathrm{m}$ of the particle diameter allowed efficient migration of bone-producing cells and colonization by blood vessels, that are essential for new bone formation ${ }^{15,16)}$. Therefore, we used IP-CHA granules of approximately $500 \mu \mathrm{m}$ diameter as a graft material. Although porous HA exhibited good osteoconduction, it did not enhance osteoinduction. Because it was fabricated at a high temperature and hardly-absorbed, $\mathrm{CaPO}_{4}$ mineral was not eluted and did not effect in vivo osteogenic differentiation ${ }^{17}$. In contrast, preadsorption of poly $(\mathrm{P})$ onto $\mathrm{HA}$ promoted osteogenic differentiation of stem cells to facilitate the formation of new bone $\mathrm{e}^{9,10,12)}$. It is considered that Poly(P)/IP-CHA has ability both osteoconduction and osteroinduction in bone regeneration.

Our previous study showed that adsorption per unit weight of IP-CHA was not significantly influenced by the block size in the range of $35.3 \mathrm{~mm}^{3}(3.0 \mathrm{~mm}$ in length, $5.0 \mathrm{~mm}$ in diameter) to $785 \mathrm{~mm}^{3}(10 \mathrm{~mm}$ in length, $10 \mathrm{~mm}$ in diameter $)^{13)}$. Thus, we speculated that poly $(\mathrm{P})$ would be uniformly adsorbed onto IPCHA granules as small as $500 \mu \mathrm{m}$. In fact, toluidine blue staining revealed that poly $(\mathrm{P})$ was adsorbed over the entire surface of these fine IP-CHA granules. We were initially concerned that the interconnected porous structure of IP-CHA would be disrupted by adsorbed poly $(\mathrm{P})$, but SEM evaluation showed no changes in the characteristic three-dimensional structure of the interconnected pores known to be critical for osteoconduction. These results coincided with that of the adsorbed poly(P) onto IP-CHA ${ }^{13)}$. Mechanical strength also did not influence the adsorption process. Thus, these Poly(P)/IP-CHA granules should have an osteoconduction capacity similar to the untreated granules IP-CHA. In order to clarify the effect of poly(P) for GBR, IP-CHA group was used as controls and we did not used autogenous bone for the animal procedure in this study.

Histology revealed bone regeneration and reconstruction of the original defect shape at implant sites using either Poly(P)/IP-CHA or IP-CHA as a grafting material, but Poly(P)/IP-CHA proved superior in other aspects. The principle outcome measure in this study was coverage of the exposed screw as assessed by the bone implant contact (BIC) and regenerated bone height value. Bone contact to the implant surface; osseointegration predicts implant stability within the surrounding marginal bone. Loss of marginal bone increases the risk of implant movement and periimplantitis. For these reasons, early osseointegration is important for long term implant stability and treatment success. While newly formed bone and fibrous tissue was observed in all implants, little newly formed tissue contacted the IP-CHA. During the early bone healing stage, the defect spaces at the interface between the implant and graft material (HA or xenogeneic bone) were filled with blood clots that were soon replaced by fibrous granulation tissue. These fibrous tissue sites gave rise to bone under stimulation by bone morphogenetic protein and other growth factors ${ }^{18,19)}$. poly $(\mathrm{P})$ appears to enhance bone regeneration by facilitating this process. The amount of poly(P) adsorbed onto IP-CHA is 41.2 $\mathrm{mg}$ poly $(\mathrm{P}) / \mathrm{mg}$ IP-CHA ${ }^{13)}$. The mechanism of adsorption of poly $(\mathrm{P})$ onto IP-CHA surface is a weak electrical bonding between the negatively-charged poly $(\mathrm{P})$ and the positively-charged hydroxyapatite. By assay for dynamic elution was shown that most of adsorbed poly $(\mathrm{P})$ was initially eluted, however, it may be released during implantation to affect bone healing because the quantity of circulation in bone defect seems to be limited. poly $(\mathrm{P})$ stimulates bFGF-induced cell proliferation and vascularization, and these effects resulted in a significantly higher BIC in the Poly(P)/IPCHA group than the IP-CHA group. A bovine-derived anorganic bone matrix such a Bio-Oss ${ }^{\circledR}$ has been reported superior bone formation and provides scaffold for osteoblasts as an alternative to autologous bone $\mathrm{e}^{20-22)}$.

Result from the bone socket defect model of dog's mandible, BIC in bovine-derived anorganic bone matrix with membrane was approximately $77.4 \%{ }^{23)}$. Our results on the $\mathrm{BIC} \%(67.9 \pm 11.8 \%)$ were similar to the ratio the dehiscence defect model.

Reconstruction of bone to its original shape is an important aspect of esthetic restoration. In this study, however, bone regeneration was detected at the upper part of the implant shoulder in the both groups, and there was no significant difference in the ratio of regenerated bone height. The reason for this observation is unclear. One possible explanation could be that the stability of the grafted area is a prerequisite for bone regeneration $^{24)}$. Hence, the use of the membrane technique should be used to stabilize graft materials and for space making and it might be speculated that the insufficient mechanical protection of the micro movement of the graft material should be carefully considered.

While bone defects in both treatment groups exhibited bone tissue and fibrous tissue on the defect surface, only the Poly(P)/IP-CHA scaffold promoted the development of new bone at the graft site. In the result of histological evaluation, bone formation was detected inside pores at bottom portion of bone defect, but upper and central portion of IP-CHAs pore was hardly observed. Our previous studies performed in the bone socket of rabbit femurs, placed IP-CHA were filled newly bone at early periods ${ }^{12,13)}$. These bone defects are able to be protected a mechanical stress and some negative factor for bone formation. Because of placed IP-CHA were surrounded by existed bone, nutritional supply from the vessel was abundance. On the other hand, bone defect of present study is dehiscence shape 
around the implant, it is consider that nutrition from surround vessel can hardly supply rather than in the femur model. For these reasons, inside of IP-CHA pore hardly observed bone formation. Therefore, further healing period might be necessary to achievement of newly formation in the pores. After a longer period of healing, it is possible that both graft material would support acceptable bone regeneration because of the osteoconduction capacity of IP-CHA. Indeed, in the previous study on a beagle dog's femur demonstrated that bone stromal cells injected with IP-CHA composite enhanced bone formation compared to IP-CHA after 4 weeks of healing, whereas there was no difference after 8 weeks $^{25}$. Nonetheless, more rapid generation of new bone by adsorption of poly $(\mathrm{P})$ onto the scaffold has several clinical advantages. For example, the accelerated healing would help stabilize the implant, restore esthetic appearance within a shorter period, and reduce the risk of infection.

Although an autogeneous bone graft is the gold standard for $\mathrm{GBR}^{26)}$, the surgery required for harvesting bone may cause pain and postoperative problems. In addition, the amount of bone available for use is limited and may be inadequate in some clinical cases $^{7,27)}$. Bovine-derived anorganic bone matrix has been demonstrated to promote osteoconduction ${ }^{28)}$, this product has not yet been approved for use implant therapy in Japan because of possible risks associated with bovine spongiform encephalopathies. poly $(\mathrm{P})$ exists widely in nature and has even been approved as a food additive by the World Health Organization. Interconnected porous calcium hydroxyapatite is made from synthetic HA; therefore, there is no risk of disease transfer after implantation using the Poly(P)/IP-CHA scaffold. Therefore, these results suggest that adsorbing poly $(\mathrm{P})$, granular Poly $(\mathrm{P}) / \mathrm{IP}-\mathrm{CHA}$ will enhance bone regeneration for implant GBR.

It is the authors' intentions to conduct further studies of comparison with autogenous bone or artificial bone in order to identify the advantage of Poly(P)/IPCHA for implants GBR.

\section{CONCLUSIONS}

Our results indicate that granule Poly(P)/IP-CHA facilitates both the formation of new bone and osseointegration with the dental implant. Further improvements in the fabrication of bone graft material will lead to even safer and more effective GBR for around dental implants.

\section{ACKNOWLEDGMENTS}

This study was supported by a Grant-in-aid for Scientific Research (No. 24592918 and No. 23390443) from the Japan Society for the Promotion of Science.

\section{REFERENCES}

1) Schropp L, Wenzel A, Kostopoulos L, Karring T. Bone healing and soft tissue contour changes following single-tooth extraction: a clinical and radiographic 12-month prospective study. Int J Periodontics Restorative Dent 2003; 23: 313323.

2) Chen ST, Wilson TG, Hämmerle CH. Immediate or early placement of implants following tooth extraction: review of biologic basis, clinical procedures, and outcomes. Int J Oral Maxillofac Implants 2004; 19: 12-25.

3) Cardaropoli G, Araujo M, Hayacibara R, Sukekava F, Lindhe J. Healing of extraction sockets and surgically producedaugmented and non-augmented-defects in alveolar ridge. An experimental study in the dog. J Clin Periodontol 2005; 32: 435-440.

4) Dahlin C, Linde A, Gottlow J, Nyman S. Healing of bone defects by guided tissue regeneration. Plastic and reconstructive surgery 1988; 81: 672-676.

5) Hämmerle $\mathrm{CH}$, Karring T. Guided bone regeneration at oral implant sites. Periodontology 2000 1998; 17: 151-175.

6) Hämmerle $\mathrm{CH}$, Jung RE. Bone augmentation by means of barrier membranes. Periodontology 2000 2003; 33: 36-53.

7) Burchardt H. The biology of bone graft repair. Clin Orthop Relat Res 1983; 174: 28-42.

8) Reuben SS, Vieira P, Faruqi S, Verghis A, Kilaru PA, Maciolek $\mathrm{H}$. Local administration of morphine for analgesia after iliac bone graft harvest. Anesthesiology 2001; 95: 390-394.

9) Shiba T, Nishimura D, Kawazoe Y, Onodera Y, Tsutsumi K, Nakamura R, Ohshiro M. Modulation of mitogenic activity of fibroblast growth factors by inorganic polyphosphate. J Biol Chem 2003; 278: 26788-26792.

10) Kawazoe $Y$, Shiba T, Nakamura R, Mizuno A, Tsutsumi K, Uematsu T, Yamaoka M, Shindoh M, Kohgo T. Induction of calcification in MC3T3-E1 cells by inorganic polyphosphate. J Dent Res 2004; 83: 613-618.

11) Tamai N, Myoui A, Tomita T, Nakase T, Tanaka J, Ochi T, Yoshikawa H. Novel hydroxyapatite ceramics with an interconnective porous structure exhibit superior osteoconduction in vivo. J Biomed Mater Res 2001; 59: 110117.

12) Yuan Q, Kubo T, Doi K, Morita K, Takeshita R, Katoh S, Shiba T, Gong P, Akagawa Y. Effect of combined application of bFGF and inorganic polyphosphate on bioactivities of osteoblasts and initial bone regeneration. Acta Biomater 2009; 5: 1716-1724.

13) Morita K, Doi K, Kubo T, Takeshita R, Kato S, Shiba T, Akagawa Y. Enhanced initial bone regeneration with inorganic polyphosphate-adsorbed hydroxyapatite. Acta Biomater 2010; 6: 2808-2815.

14) Kubo T, Doi K, Hayashi K, Morita K, Matsuura A, Teixeira ER, Akagawa Y. Comparative evaluation of bone regeneration using spherical and irregularly shaped granules of interconnected porous hydroxylapatite. A beagle dog study. J Prosthodont Res 2011; 55: 104-109.

15) Wang HL, Pappert TD, Castelli WA, Chiego DJ Jr, Shyr Y, Smith BA. The effect of platelet-derived growth factor on the cellular response of the periodontium: an autoradiographic study on dogs. J Periodontol 1994; 65: 429-436.

16) Anderegg CR, Alexander DC, Freidman M. A bioactive glass particulate in the treatment of molar furcation invasions. $J$ Periodontol 1999; 70: 384-387.

17) Harris CT, Cooper LF. Comparison of bone graft matrices for human mesenchymal stem cell-directed osteogenesis. J Biomed Master Res 2004; 68A: 747-755.

18) Tabata Y, Yamada K, Miyamoto S, Nagata I, Kikuchi H, Aoyama I, Tamura M, Ikada Y. Bone regeneration by basic fibroblast growth factor complexed with biodegradable hydrogels. Biomaterials 1998; 19: 807-815

19) Jeon O, Song SJ, Yang HS, Bhang SH, Kang SW, Sung MA, Lee JH, Kim BS. Long-term delivery enhances in vivo osteogenic efficacy of bone morphogenetic protein-2 compared 
to short-term delivery. Biochem Biophys Res Commun 2008: 369; 774-780.

20) McAllister BS, Margolin MD, Cogan AG, Taylor M, Wollins J. Residual lateral wall defects following sinus grafting with recombinant human osteogenic protein-1 or Bio-Oss in the chimpanzee. Int J Periodontics Restorative Dent 1998; 18: 227-239.

21) Hoffman S, Sidqui M, Abensur D, Valentini P, Missika P. Effects of Laddec on the formation of calcified bone matrix in the rat calvariae cells culture. Biomaterials 1999; 20: 155166.

22) Stephan EB, Jiang D, Lynch S, Bush P, Dziak R. Anorganic bovine bone supports osteoblastic cell attachment and proliferation. J Periodontol 1999; 70: 364-369.

23) Botticelli D, Berglundh T, Lindhe J. The influence of a biomaterial on the closure of a marginal hard tissue defect adjacent to implants. An experimental study in the dog. Clin Oral Implants Res 2004; 15: 285-292.
24) Wikesjö UM, Nilvéus R. Periodontal repair in dogs: effect of wound stabilization on healing. J Periodontol 1990; 61: 719724.

25) Doi K, Kubo T, Hayashi K, Imura K, Akagawa Y. Development of cell-hybridized artificial bone: Effect of osteogenic differentiation of bone marrow stromal stem cells on bone formation with newly developed interconnected porous hydroxyapatite. Dent Mater J 2007; 26: 162-169.

26) Misch CM, Misch CE. The repair of localized severe ridge defects for implant placement using mandibular bone grafts. Implant Dent 1995; 4: 261-267.

27) Nasr HF, Aichelmann-Reidy ME, Yukna RA. Bone and bone substitutes. J Periodontol 1999; 19: 74-86.

28) Klinge B, Alberius $\mathrm{P}$, Isaksson S, Jörgenm J. Osseous response to implanted natural bone mineral and synthetic hydroxylapatite ceramic in the repair of experimental skull bone defects. J Oral Maxillofac Surg 1992; 50: 241-249. 